Cad.Est.Ling., Campinas, (41):7-35, Jul./Dez. 2001

\title{
GESTION DU TOPIC ET ORGANISATION DE LA CONVERSATION
}

\author{
LORENZA MONDADA \\ Université de Bâle \& Université de Lyon 2
}

RÉSUMÉ: Cet article développe une approche de la notion de "topic" dans la perspective de l'analyse conversationnelle d'origine ethnométhodologique. Cette approche est caractérisée par trois préoccupations spécifiques: en premier lieu, elle traite la gestion du topic par rapport à la question générale de l'organisation de la parole en interaction et à la question particulière de la façon dont celle-ci est assurée par les locuteurs en exploitant de façon située les ressources linguistiques; en deuxième lieu, elle insiste sur le caractère "endogène" de la définition du topic, en décrivant la façon dont les locuteurs eux-mêmes la traitent à toutes fins pratiques dans leurs activités interactionnelles; en troisième lieu, elle aborde l'organisation topicale moins du point de vue des formes linguistiques utilisées pour la marquer que du point de vue des procédés interactifs mis en oeuvre par les participants, pouvant exploiter localement des formes linguistiques spécifiques.

\section{INTRODUCTION}

\subsection{Objectifs}

Ce texte vise à expliciter les apports spécifiques de l'analyse conversationnelle d'inspiration ethnométhodologique à des problèmes classiquement catégorisés en linguistique comme relevant de la "référence", de la "référenciation", de la "topicalité", des "objets de discours". Pour ce faire, il précise d'abord la "mentalité analytique" de la linguistique interactionnelle pratiquée ici, pour ensuite développer quelques propriétés de ces objets de discours particuliers que sont les topics.

\subsection{Horizon conceptuel: la linguistique interactionnelle}

Cette contribution ${ }^{1}$ se fonde sur la linguistique interactionnelle, entendue ici comme le tournant linguistique de l'analyse conversationnelle d'inspiration ethnométhodologique. Il existe en effet de nombreuses approches de l'interaction dans le paysage des sciences du langage, qui se basent sur des présupposés différents et des cadres théoriques distincts, allant de l'analyse des faces de Goffman à l'analyse du

\footnotetext{
${ }^{1}$ Pour une version brève de ce texte, on lira Mondada (à paraître a).
} 
discours de l'Ecole de Birmingham; parmi elles l'analyse conversationnelle au sens strict, d'inspiration ethnométhodologique, initiée par Harvey Sacks dans les années 70 (cf. Sacks, 1992) et prolongée dans sa collaboration avec Schegloff (cf. Sacks, Schegloff, Jefferson, 1974), constitue une tendance spécifique (cf. les distinctions que fait Levinson, 1983, ch. 6; cf. Gülich \& Mondada, à paraître pour une présentation détaillée).

Alors que Sacks et ses collègues (cf. Schegloff \& Sacks, 1973) ne visaient pas à l'origine une analyse du langage mais plutôt une analyse des activités sociales que les enregistrements (limités à cette époque à l'audio) rendaient disponibles pour une écoute répétée et donc pour une analyse détaillée, ces dernières années l'analyse conversationnelle a progressivement pénétré au sein de plusieurs secteurs de la linguistique (cf. Ochs, Schegloff, Thompson, 1996). Sa réception, néanmoins, reste ambiguë: si d'une part de nombreux linguistes reconnaissent l'efficacité de l'analyse conversationnelle pour le traitement des données orales interactives, d'autre part ils la réduisent souvent à un ensemble d'outils techniques et résistent ainsi à adopter la "mentalité analytique" qui la caractérise (cf. Sacks, 1984; Schenkein, 1978). Cette "mentalité" implique en effet un regard particulier sur les ressources langagières: elle ne traite pas des formes pour en faire une description générale dans une grammaire, mais plutôt des procédés des locuteurs (les "ethnométhodes", Garfinkel, 1967) mis en oeuvre pour construire le sens, assurer l'intercompréhension, rendre possible les activités langagières ordinaires. Elle ne traite pas de catégories descriptives abstraites dont les conditions d'application seraient définies de façon nécessaire et suffisante et qui constitueraient un système que les locuteurs ne feraient qu'actualiser, mais de catégories rendues localement pertinentes par les orientations situées des locuteurs dans leurs activités spécifiques. Elle ne traite pas d'unités minimales définies a priori par leurs caractéristiques structurales et combinables selon des règles prédéfinies, mais d'unités plastiques, dynamiques, émergentes, qui se constituent dans la temporalité des séquences interactionnelles et dans les ajustements entre les locuteurs et qui donc sont contingentes par rapport à leurs actions (Goodwin, 1979; Ford, 1993; Selting, 1995; Ono \& Thompson, 1995; Mondada, 1995a, 2000a, à paraitre b).

Il découle de cette approche spécifique une vision particulière de la langue: la langue, en effet, existe d'abord dans et par les pratiques langagières des locuteurs; elle est profondément imbriquée en elles et ne peut donc être définie indépendamment d'elles: il s'agit de se demander comment la langue se manifeste et se constitue en même temps dans les pratiques, c'est-à-dire, plus précisément, comment les pratiques langagières, et notamment les activités interactionnelles des participants, identifient, exploitent, et par là configurent les ressources de ce qui sera désigné (par eux, par les entreprises normatives, par les grammaires) comme étant la langue. La langue appartient aux locuteurs - avant qu'au linguiste; c'est le "je" qui se la réapproprie dans chaque acte d'énonciation, qui la réinvente pour mieux s'ajuster à la situation en cours. La codification et la standardisation ne sont donc pas les seuls aspects définissant la langue; elles sont le résultat de pratiques sédimentées, à décrire dans leurs effets constituants et non pas à considérer dans leur évidence constituée (Mondada, 1995a; à paraître b). 


\subsection{Référence, référenciation, objets de discours et topics}

Dans ce cadre, nous allons nous pencher sur les phénomènes qui relèvent de débats désormais classiques dans la littérature concernant les dimensions référentielles et thématiques des activités des locuteurs.

La question de la référence est un thème classique de la philosophie du langage, de la logique et de la linguistique; dans ces cadres elle a été historiquement posée comme un problème de représentation du monde, de verbalisation du référent où la forme linguistique choisie est évaluée en termes de vérité et de correspondance avec lui. La question de la référenciation opère un glissement par rapport à ce premier cadre: elle ne privilégie pas la relation entre les mots et les choses, mais la relation intersubjective et sociale au sein de laquelle des versions du monde sont publiquement élaborées, évaluées en termes d'adéquation aux finalités pratiques et aux actions en cours des énonciateurs (Mondada \& Dubois, 1995). De cette façon, ce sont les procédés mis en oeuvre par les participants à l'interaction pour assurer des opérations de référenciation qui deviennent l'objet de la description de l'analyste.

$\mathrm{Au}$ sein de ces opérations de référenciation, les interlocuteurs élaborent des objets de discours, i.e. des entités qui ne sont pas conçues comme des expressions référentielles en relation spéculaire avec des objets du monde ou avec leur représentation cognitive, mais des entités qui sont interactivement et discursivement produites par les participants au fil de leur énonciation. Les objets de discours sont donc des entités constituées dans et par les formulations discursives des participants: c'est dans et par le discours que sont posés, délimités, développés et transformés des objets de discours qui ne lui préexistent pas et qui n'ont pas une structure fixe, mais qui au contraire émergent et s'élaborent progressivement dans la dynamique discursive. Autrement dit, l'objet de discours ne renvoie pas à la verbalisation d'un objet autonome et externe aux pratiques langagières; il n'est pas un référent qui aurait été codé linguistiquement (Mondada, 1994).

Certains de ces objets de discours peuvent assumer un statut particulier dans le discours et dans l'interaction - identifié, reconnu et défini comme tel par les partipants eux-mêmes - et être ainsi traités comme des topics, i.e. des objets considérés et manifestés par les participants comme ce à propos de quoi ils énoncent le discours en train de se faire. Contrairement à ce qui est souvent le cas dans la littérature abondante consacrée à ce domaine, le topic est ainsi conçu dans ce cadre non pas comme une catégorie savante définie par le linguiste sur la base de critères analytiques, mais plutôt comme une entité pertinente pour les locuteurs, qui lui attribuent des propriétés spécifiques aux fins pratiques de leur action interactionnelle.

Dans ce qui suit, nous allons donc nous concentrer sur un certain nombre de propriétés des topics qui ne sont que très partiellement considérées par les approches grammaticales et cognitives de la référence et qui jouent par contre un rôle central dans l'approche interactionnelle découlant de l'analyse conversationnelle. Nous insisterons en particulier sur le rapport entre organisation de la conversation et gestion interactionnelle du topic (2.), sur les propriétés que les locuteurs attribuent en contexte aux topics dont ils parlent (3.), sur les procédés par lesquels ils définissent des configurations topicales spécifiques (4.), en collaborant interactionnellement à leur établissement et transformation (5.), notamment grâce à une exploitation située des 
ressources langagières (6.). Nous verrons ainsi que la formulation du topic est accomplie en mobilisant, dans des environnements séquentiels particuliers, ce qu'on pourrait appeler la grammaire-en-action, i.e. un ensemble de ressources indexicalement appropriées à l'activité conversationnelle.

\section{L'ORDRE DE LA CONVERSATION COMME ACCOMPLISSEMENT INTERACTIONNEL}

\subsection{Une perspective conversationnelle sur le topic}

Nous allons donc ici développer les apports de l'analyse conversationnelle d'inspiration ethnométhdologique à la problématique du topic dans l'interaction ${ }^{2}$. De nombreuses questions se posent en effet lorsqu'on se penche sur les activités topicales des locuteurs: comment rendre compte du fait que la conversation est dotée d'un ordre et d'une intelligibilité pour les participants? comment est-ce que les locuteurs produisent des apports conversationnels thématiquement pertinents? comment accomplissent-ils leur parler thématique, non seulement en alimentant la conversation avec des topics, mais encore en conversant de façon thématiquement pertinente? comment mobilisent-ils de façon locale et indexicale les ressources de la langue aux fins des placements et des négociations topicales?

Bien qu'au premier abord une transcription puisse donner l'impression d'un fourmillement chaotique de bribes et d'hésitations, d'orientations et de trajectoires possibles, la conversation est un phénomène ordonné, dont l'ordre est à la fois le principe de l'intelligibilité de l'événement et le résultat des activités de coordination des locuteurs. Bien que le propre de la conversation spontanée soit la non-programmation des topics qui y sont abordés, son analyse révèle des cohérences, des modes d'organisation particuliers régissant les contributions thématiques. Et bien que les interactions formelles soient souvent caractérisées par une programmation des topics (par un ordre du jour, par exemple), sa réalisation est le fait d'un accomplissement collectif situé des participants, qui en tant que tel n'est pas déterminé par l'agenda thématique mais se réalise indexicalement. Ces quelques remarques suffisent à évoquer la complexité des problèmes soulevés par l'analyse du topic dans la conversation, qui présente à la fois l'intérêt et la difficulté d'être un terrain où il faut tenir compte des dynamiques, des transformations, des variations indexicales constitutives de l'objet étudié.

Ces remarques esquissent aussi un ensemble de questions par lesquelles il est possible de reformuler le problème du topic du point de vue de l'analyse conversationnelle d'inspiration ethnométhodologique. Nous allons donc nous centrer d'abord sur la façon dont cette perspective permet de reposer la question de l'organisation des topics dans la conversation et de se doter d'outils d'analyse adéquats pour y répondre. Cette approche permet notamment de rendre compte du fait que le topic constitue un "phénomène" pour les locuteurs, un problème pratique traité par les participants, et que, dans ce sens, il n'est pas d'abord une construction de l'analyste.

\footnotetext{
${ }^{2}$ Voir pour une présentation complémentaire, axée sur d'autres phénomènes, Mondada (1995b).
} 


\subsection{La notion d'accomplissement interactionnel}

Une des caractéristiques majeures de l'analyse conversationnelle d'inspiration ethnométhodologique est de se centrer sur les activités sociales, et notamment langagières, des acteurs en les concevant non pas comme guidées par des règles, des normes, des schémas sousjacents et préexistants, mais comme des processus endogènes, accomplis collectivement par les participants, élaborés localement dans leur ajustement mutuel et dans leur adéquation au contexte. La notion d'accomplissement est ainsi centrale $^{3}$ : elle permet de rendre compte des actions, mais aussi des faits, des événements, des représentations, comme étant réalisés grâce à la mise en oeuvre de procédures dont la caractéristique est de s'auto-organiser dans le contexte de cette mise en oeuvre. Ces procédures sont ordonnées, et leur ordre n'est pas assuré par l'intériorisation de valeurs ou d'habitudes, mais par le déploiement mutuel des orientations des acteurs envers le contexte et l'action: en rendant disponible, accessible, descriptible (accountable) son action à son interlocuteur, le locuteur la dote d'une intelligibilité qui permettra au locuteur suivant de s'y ajuster, d'y répondre, d'en modifier ou d'en élaborer le sens ${ }^{4}$.

Cette approche est particulièrement intéressante pour aborder les dynamiques thématiques dans l'interaction verbale, puisque celles-ci ne sont pas la plupart du temps prévisibles, comme c'est le cas des réunions avec un agenda thématique, mais se caractérisent par le fait que des topics émergent et se transforment constamment, sont abandonnés, repris, négociés, maintenus, défendus, concurrencés, etc. La question se pose alors de comment suivre ces trajectoires complexes, comment y identifier un ordre, comment reconnaître l'intelligibilité que leur prêtent les locuteurs eux-mêmes. La réponse se penche sur les activités thématiques des locuteurs plutôt que sur la structuration sémantique des topics qu'ils énoncent: les dynamiques thématiques rendent observables les procédures que les locuteurs mettent en oeuvre pour introduire un nouveau topic lorsque la conversation s'essoufle, pour insérer un topic particulier dans un développement thématique déjà établi, pour contrecarrer le développement thématique d'un autre participant, etc. L'accent mis sur les procédures permet de donner un traitement formel à une problématique qui autrement risquerait d'être abordée en termes de contenus - ce risque fondant par ailleurs la méfiance des analystes de la conversation envers ce domaine d'enquête (cf. Sacks, 1992, I, 752; Schegloff, 1990, 51-2). Ces procédures, en outre, ne sont pas le fait d'un locuteur individuel, décidant en solitaire des mouvements à faire, mais sont constitutivement interactives, étant orientées vers les autres participants (recipient designed) et étant déployées tour par tour, de façon sensible aux enchaînements séquentiels précédents et suivants. Une fois introduits dans la conversation — cette introduction étant elle-même à négocier les topics n'appartiennent plus en propre à celui qui les a proposés, mais sont

\footnotetext{
${ }^{3}$ Cf. l'ouvrage fondamental de Garfinkel (1967), ainsi que les introductions de Heritage $(1984,1992)$ et, en français, de Coulon (1993).

${ }^{4}$ Nous ne pouvons pas faire ici une synthèse des principes de l'analyse conversationnelle. On pourra consulter utilement pour une introduction: Bange (1992), Kallmeyer \& Schutze (1976), Gulich (1990), Levinson (1983, ch. 6), Psathas (1995), et plus récemment ten Have (1998), Hutchby \& Wooffitt (1998), et Gülich \& Mondada (à paraître). Voir en portugais Marcuschi (1998).
} 
configurés à travers l'activité collective des participants. Le topic d'une séquence ou d'une conversation entière est donc accompli interactivement au fil de l'interaction par les participants.

Pour cadrer cette description des procédures thématiques mises en oeuvre par les locuteurs, nous allons d'abord situer leur déploiement tel qu'il s'organise au fil de la séquentialité de la conversation, qui prend forme dans l'alternance des tours de parole (2.3.) et dans sa structuration en unités séquentielles minimales, les paires adjacentes (2.4.), qui peuvent être exploitées pour insérer et contrôler la forme des topics (3ss). Ces structures ne relèvent pas d'un modèle abstrait, mais sont reconnues et rendues reconnaissables par les locuteurs eux-mêmes, qui s'y orientent pour rendre adéquate leur contribution thématique.

\subsection{Les tours de parole comme structure ordonnée élémentaire}

La question du caractère ordonné des activités sociales, telles que les acteurs eux-mêmes les organisent dans le détail de leur accomplissement, s'est située au coeur de la problématique de l'analyse de la conversation depuis ses débuts - identifiables avec les leçons de Harvey Sacks durant les annces 60, publiées récemment (1992), et l'article princeps qu'il écrivit avec Emmanuel Schegloff et Gail Jefferson (1974) sur la "machinerie" des tours de parole.

Les tours de parole sont le fondement de l'ordre conversationnel dans la mesure où ils permettent d'observer les processus élémentaires de coordination et de synchronisation de l'action conjointe par les participants. Ceux-ci, en effet, doivent affronter un problème pratique: comment organiser leurs prises de parole de sorte à éviter que se produisent à la fois des chevauchements, lorsque plusieurs personnes parlent en même temps, et des silences, lorsque personne ne prend la parole. Ce problème est résolu par les participants avec une remarquable précision, grâce à deux types de procédures qui organisent l'alternance des tours de parole: d'une part les techniques d'allocation de la parole, d'autre part les méthodes d'identification des lieux de transition possibles où l'alternance peut prendre place.

Les premières permettent de distinguer les procédés d'auto-sélection, par lesquels un locuteur prend la parole et s'attribue le tour, des procédés d'hétéro-sélection, par lesquels le locuteur suivant est désigné par celui qui a la parole. Le deuxième ensemble de techniques rend compte de la capacité qu'ont les locuteurs de repérer méthodiquement et systématiquement les points de transition potentiels de la parole: ils se les rendent mutuellement reconnaissables grâce à des procédures d'interprétation et de production qui exploitent, de façon située et au fil du déroulement temporel des énoncés, des formes linguistiques et non-linguistiques. Les énonciateurs comme les énonciataires effectuent ainsi une analyse en temps réel de l'élaboration du tour de parole, de sorte à projeter sa fin possible, que ce soit, du côté du locuteur, pour maintenir ou pour passer la parole, ou, du côté de son interlocuteur, pour la laisser ou la prendre (Ochs, Schegloff, Thompson, 1996; Mondada, 2001).

Les mécanismes qui permettent de passer d'un tour à l'autre ne sont pas sensibles au type de topic qui y est traité, mais ils fournissent à la conversation sa cohérence de base, tour par tour. Ils intéressent les dynamiques thématiques au moins de deux façons, qui posent deux types de problèmes par rapport à la production d'un flux discursif 
interactionnel continu. D'une part, un changement de topic, et donc l'introduction d'un nouveau topic, peut fonctionner comme une solution à une discontinuité ou à un ralentissement du flux interactionnel et donc comme une ressource pour relancer l'alternance régulière des tours, par exemple lorsque les pauses se multiplient et le passage de la parole devient problématique (Maynard, 1980). D'autre part, lorsque le flux interactionnel est continu et régulier, les enchaînements d'un tour à l'autre peuvent être le lieu d'un travail collaboratif sur le topic:

Extrait 1 (lsun91)

$\begin{array}{lll}1 & \text { A } & \text { parce que lui i m'dit moi mes fournisseurs ils } \\ 2 & & \text { viennent hein et: } \\ 3 & \text { B } & \text { ouais c'est ça } \\ 4 & \text { A } & \text { puis j'peux pas leur dire on m'a payé hein [xxx } \\ 5 & \text { B } & \\ 6 & & \text { après tu as plus rien } \\ 7 & \text { A } & \text { mhm mhm } \\ 8 & \text { B } & \text { c'est c'est chez nous la même chose tu vois et } \\ 9 & & \text { souvent on donne encore des bons: rabais ou comme } \\ 10 & & \text { ça et puis ((rit)) } \\ 11 & \text { A } & \text { ouais } \\ 12 & \text { B } & \text { y a cet argent qui rentre pas euh } \\ \text { 13 } & \text { A } & \text { mhm mhm }\end{array}$

Le type d'enchaînement réalisé par les locuteurs d'un tour à l'autre peut exhiber un accord sur le topic: ici $\mathrm{B}$ enchaîne sur l'énoncé de $\mathrm{A}$ en 5 , en produisant la deuxième partie d'une argumentation, qui s'appuie sur le tour précédent pour le compléter. Ensuite, dans un deuxième mouvement, B garde la parole (8-10) pour apporter un exemple développant le même topic ("la même chose"), en se recentrant sur son propre cas ("chez nous"), qu'il développe dans le sens d'une généralisation ("souvent on donne"). Dans cet exemple, la continuité thématique est assurée au moyen de la continuité syntaxique, même s'il y a un changement de locuteur, puisque $B$ en complétant l'énoncé de A prend la parole et la garde $(5 \mathrm{sv})$. Le développement du topic est donc accompli dans un travail de collaboration qui dépasse l'orientation mutuelle vers le même objet, et qui concerne aussi une analyse commune et en temps réel menée conjointement par les énonciateurs sur le détail des structures syntaxiques qu'ils produisent (Mondada, 1999). Cette analyse peut être favorisée, comme c'est le cas ici, par l'utilisation de formats syntaxiques complétables, qui projettent dans le cours de leur énonciation la forme possible de leur composant final et donc projettent séquentiellement la possibilité qu'un locuteur autre que celui qui a initié l'énoncé puisse le terminer (Lerner, 1991).

En enchaînant sur la parole de son interlocuteur, chaque locuteur déploie ainsi son intelligence du tour précédent. Dans le passage d'un tour et l'autre, en effet, se gère l'intercompréhension des participants, dans un jeu de relations à la fois prospectives et rétrospectives. Chaque tour exerce sur le suivant des contraintes qui réduisent les mouvements possibles en cette position; en retour le deuxième tour manifeste l'interprétation à toutes fins pratiques qui est faite du premier tour et en ce sens exerce 
un effet rétrospectif sur lui, en contribuant à le configurer et à lui donner un sens. Ainsi se crée entre les deux un espace d'intercompréhension, qui ne relève pas d'une compréhension décontextualisée et abstraite de l'événement mais qui est plutôt une compréhension située, orientée vers les buts et les visées argumentatives des participants. Le premier locuteur a d'ailleurs la possibilité d'intervenir sur les effets rétrospectifs exercés par le second locuteur, en pouvant, s'il le juge nécessaire, réparer l'interprétation qui a été donnée de ce qu'il a dit: dans le troisième tour, le premier locuteur procèdera alors à une réparation qui réaffirme ce dont il était question et ce qu'il fallait éventuellement en retenir ou en inférer".

La réparation peut intervenir pour rétablir les malentendus, les pannes, les troubles les plus divers, parmi lesquels on trouve aussi les difficultés de gestion du topic. En effet celle-ci est plus généralement associée à la construction de la relation interactionnelle, de l'intercompréhension et du savoir intersubjectif des locuteurs et est plus particulièrement liée à la production d'enchaînements entre un tour et l'autre et donc aux attentes, contrôles et interprétations propres aux dynamiques prospectives et rétrospectives, comme on peut l'observer dans l'extrait suivant:

\section{Extrait 2 (ciu.imru315-319)}

1 M aujourd'hui/ le quartier se compartimente/ . c'est-à-

2 dire $\backslash$ il reste mélangé/ .. mais/ il est il est

3 compartimentél vous avez les homosexuels la nuit/.

4 les juifs le jourl. euh: vous avez des gens de: .

5 toutes sortes d- d'origines/ qui sont là/ mais qui ne

6 se fréquentent PAS\. qui se cotoient/ . dans les

7 rues/

8 E les sépharades ne fréquentent pas les ashkenazes/.

$9 \quad$ [si:

10 M [si si si si si vous avez la librairie du progrès/

11 qui est un . polonais ((rit))

12 E bien sûr

13 M comme on n'en fait plus/

14 E ah

$15 \mathrm{M}$ enfin un juif d'Europe centrale/ xxxxx un vrai de

16 vrai/ . bon qui est là/ et qui TROne dans la rue/

17 avec l'épicier/ avec xxx/machin etcaetera/ tout ça

18 ça se fréquente bien sûr (hh) . mais euh:: je parle

19 des . des non-juifs justement/ qui viennent euh: .

20 et qu'on ne voit PAS/ ils sont là/ . j'ai appris leur

21 existence parce que j'étais placé au troisième

22 étage/ . n'est-ce pas/. mais . je les ai jamais vus

23 dans la rue/

L'enchaînement que E produit à la description de A (en 8) exhibe à la fois la compréhension que $\mathrm{E}$ effectue du tour précédent et la sélection que celle-ci opère par

\footnotetext{
${ }^{5}$ Pour la notion de réparation voir Schegloff, Jefferson \& Sacks (1977), Schegloff (1979; 1991).
} 
rapport au topic proposé. Alors que A (1-7) distingue plusieurs parties (en opposant juifs et homosexuels), E (8) n'en retient qu'une (les juifs), qu'il considère à son tour comme un tout clivé (les juifs s'opposant en séfarades et ashkénazes). Le problème est soulevé par le fait que l'affirmation de A, portant sur le cloisonnement des populations, s'applique bien à l'ensemble qu'il a spécifié mais pas à celui que retient E. A est d'accord avec E sur le fait que la partition effectuée par ce dernier ne répond pas à sa caractérisation des rapports sociaux dans son premier tour, mais se trouve en même temps dans la situation de devoir rectifier ce sur quoi portait sa propre description. Il se trouve donc à devoir effectuer deux opérations, l'une d'accord et l'autre de désaccord, qui ne peuvent se faire en même temps; si la première peut enchaîner immédiatement sur ce que dit $\mathrm{E}$ (en 10 jusqu'à 18), la deuxième doit réintroduire les données initiales (par le connecteur "mais" qui réoriente l'argumentation et par le marqueur métalinguistique "je parle" 18) par des marqueurs d'autant plus explicites que le tour de parole auquel ils se rattachent un lien est rendu distant par la succession de nouveaux tours de parole.

Cet exemple rend manifestes à la fois les difficultés et les enjeux de l'explicitation ou de la contestation de la compréhension, souvent liée à la configuration d'un domaine thématique. Il montre la difficulté pour le locuteur de remonter le cours de la temporalité séquentielle de l'interaction pour élaborer ou rétablir un élément mal compris ou focalisé à tort; il montre aussi comment les processus de compréhension émergent de façon observable dans la séquentialité de l'interaction. En effet, la compréhension n'est pas un processus cognitif abstrait qui se déroulerait dans les arcanes de l'esprit et qui serait par conséquent inaccessible à l'observation, mais une production interactionnelle qui se manifeste notamment dans l'enchaînement par lequel le locuteur suivant prend appui sur les dires du locuteur actuel et dans lequel il manifeste ce qu'il en retient. La compréhension ainsi rendue publiquement disponible peut être tacitement acceptée ou bien réfutée ou réorientée, par une intervention réparatrice, comme c'est le cas ici.

\subsection{Séquentialité des paires adjacentes et organisation thématique}

Les relations entre deux tours de parole sont particulièrement étroites lorsqu'ils forment une paire adjacente: par ce terme Sacks et Schegloff (1973) renvoient à deux tours produits par deux locuteurs différents, qui constituent l'un la première partie de la paire et l'autre la seconde, qui sont ordonnés et qui s'enchaînent immédiatement. Les couples de salutations, l'articulation entre question et réponse, entre proposition et acceptation (ou refus) constituent des exemples de paires adjacentes. Ce qui les caractérise est leur caractère reconnaissable et contraignant: l'apparition de la première partie suscite des attentes normatives quant à l'apparition de la seconde. Entre les deux se noue ainsi une relation d'implicativité séquentielle ${ }^{6}$.

La relation étroite qui s'établit entre les paires adjacentes est un principe fondamental de construction de la cohérence de la conversation. Elle joue donc un rôle crucial pour la gestion du topic, même si celle-ci ne s'y réduit pas. On peut en effet

\footnotetext{
${ }^{6}$ Pour la définition originale de la notion de paire adjacente, voir Sacks \& Schegloff (1973, 295sv). Pour une présentation en français, voir Bange (1992, 39sv).
} 
utiliser le mécanisme de la paire adjacente pour contraindre le développement thématique: on peut par exemple poser une question pour introduire ou faire introduire un topic dont on veut parler (Sacks \& Schegloff, 1973, 296). Toutefois cette exploitation de l'implicativité séquentielle a elle-même des contraintes et produit un développement conversationnel structurellement spécifique, distinct d'autres modes séquentiels d'organisation thématique (Schegloff, 1990). Ainsi Sacks (1992, II, 565) distingue les effets possibles sur le traitement thématique de l'organisation d'une séquence en paires adjacentes et de l'organisation d'une séquence autour d'un topic. Dans ce dernier cas, on a l'introduction d'un topic et un accord autour de son développement, permettant son élaboration dans une séquence éventuellement étendue. Dans le premier cas, par contre, on a une première partie de paire, par exemple une question, imposant un topic, suivie d'une seconde partie, par exemple une réponse, le reprenant: le développement thématique risquera de se terminer avec la clôture de la paire adjacente, et de ne pas aller au-delà, la réponse pouvant signifier "Je ne vais pas en parler plus longuement maintenant" ou "On en reparlera plus tard".

Il y a ainsi des développements thématiques qui se limitent à des enchaînements de paires adjacentes sans donner lieu à des élaborations plus étendues: c'est souvent le cas des conversations où les modes d'intervention sont distribués spécifiquement et asymétriquement parmi les participants comme dans l'exemple suivant:

$\begin{array}{ccl}\text { Extrait } 3 & \text { (ESF/BE19A-5) } \\ 1 & \text { H } & \text { vous aimez la musique/ } \\ 2 & \text { B } & \text { oui } \\ 3 & \text { H } & \text { quelle musiques tu aimes/ } \\ 4 & \text { B } & \text { euh romantique } \\ 5 & \text { H } & \text { romantique } \\ 6 & \text { B } & \text { oui ((rit)) } \\ 7 & \text { H } & \text { c'est-à-dire qu'est-ce que tu aimes/ } \\ 8 & \text { B } & \text { euh beaucoup de gars de espagnol } \\ 9 & \text { H } & \text { hm } \\ 10 & \text { B } & \text { Roberto Carlo }\end{array}$

Dans cet extrait de conversation exolingue, où les deux locuteurs n'ont pas la même compétence dans la langue de l'échange, le topic est systématiquement introduit par le locuteur natif, $\mathrm{H}$, et reçoit une réponse, souvent minimale, du locuteur non-natif, B. Cette situation peut être rapprochée d'autres cadres communicationnels présentant des formats interactifs similaires, comme les situations d'apprentissage (Berthoud \& Mondada, 1992) ou certains dialogues adulte-enfant (Hudelot, 1988), où les interventions de l'adulte se spécialisent en questions (et en évaluations des réponses données) contrôlant l'introduction des topics et l'allocation des tours de parole. C'est le cas aussi des entretiens et des interviews journalistiques, où la préallocation des tours spécialise en principe l'intervieweur dans le contrôle des transitions thématiques en l'interdisant aux interviewés - bien que ceux-ci aient des stratégies pour échapper à l'enchaînement rigoureux des questions et des réponses (Greatbatch, 1986, Nofsinger, 1988/89). 
La forme structurelle que prend l'introduction du topic marque ainsi une préférence pour une réponse brève ou longue et est ainsi susceptible de jouer ou non le rôle d'une ouverture thématique. De cette façon, on peut dire que l'analyse en paires adjacentes n'est pas suffisante pour rendre compte des élaborations thématiques des participants. Celles-ci concernent des séquences plus étendues, et éventuellement non adjacentes (comme c'est le cas pour des interventions qui "recyclent" des topics apparus précédemment dans la conversation et momentanément abandonnés). D'où l'importance d'analyser les séquences spécifiquement configurées pour introduire des topics de façon durable.

\section{LA DÉFINITION LOCALEMENT SITUÉE DU TOPIC}

\subsection{L'orientation des locuteurs vers le placement adéquat du topic}

Que les interlocuteurs traitent d'un topic dans une paire adjacente ou dans un développement plus long, se pose pour eux constamment la question de savoir quand et comment placer le topic dont ils veulent parler. En effet, bien que les participants aient souvent des choses à dire quand ils se joignent à une conversation, ils n'en parlent pas n'importe où: ils ne les introduisent pas dès qu'ils ont l'occasion de parler, ni ne les délivrent en vrac au début de la conversation.

On peut donc dire que les participants s'orientent vers le fait que la conversation est thématiquement ordonnée et organisée - et ils le respectent (Sacks, I, 535). De façon plus spécifique, ceci se matérialise dans la sensibilité des participants à des problèmes de placement, tout apport conversationnel étant soumis implicitement ou explicitement à la question: "pourquoi cela maintenant et à moi?" ("why that now? "). Cette question ne se pose pas uniquement par rapport aux topics de la conversation: en contraignant leur placement adéquat, elle concerne l'organisation de la conversation tout entière.

Cette orientation vers la question "why that now?" relève aussi bien d'une interprétation rétrospective (extr. 5) des tours de parole que d'une anticipation prospective (extr. 4) de ce qui va suivre, comme dans les extraits suivants:

\section{Extrait 4 (cité par Mondada \& Zay 1999:403) (radio)}

et cette civilisation du cerf CONtinue à les imprégner au point que sous DROgue et ça c'est un thème qui reviendra: euh . tout à l'heure . sous drogue/ ils revoient l'animal qui a été la base de la civilisation de leurs ancêtres

Extrait 5 (cité par Orletti 1989:81)

1 A Bello sto maglione. Te l'ha fatto tua madre?

2 B No, l'ho comprato. E fatto a mano, però, è pure caro. Sai

3 stamattina ho comprato un barattolo di marmellata di ribes=

4 A $=$ Che vuoi dire perchè tua madre non te l'ha fatta ((ride))

5 B No, mi è venuto in mente pensando a cose che costano caro.

6 Pensa, 4500 per un barattolo piccolo=

7 A $=$ Ah adesso ti seguo, non è che fosse cosi chiaro, prima,

8 il ragionamento 
Dans l'extrait 4, le locuteur identifie explicitement le thème de son propos et en projette la pertinence vers la suite, créant ainsi à la fois des attentes et des effets de cohésion. Dans l'extrait 5, à la ligne 4, A interroge rétrospectivement la pertinence de l'enchaînement thématique que vient d'effectuer $\mathrm{B}$ en passant du pullover à la confiture: A manifeste sa propre lecture de l'enchaînement, en privilégiant un lien de continuité thématique possible, reliant les "choses faites par la mère de B" - alors que B montrera (5-6) qu'un autre lien thématique est à l'oeuvre, reliant les "choses qui coûtent cher". Ils montrent ainsi le fait que les locuteurs, de façon éventuellement divergente, recherchent et construisent constamment des pertinences thématiques. Cet échange de remarques montre que les classes thématiques sont établies ad hoc et selon les occasions conversationnelles, ne correspondant en rien à des classes sémantiques abstraites dotées de relations immanentes de similarité. C'est dans ce cadre que les raisons post-hoc peuvent être nécessaires (ce que Orletti, 1989, 77 appelle des post-hoc accountings).

\subsection{L'orientation des participants vers le caractère "connu" du topic}

La description des orientations des locuteurs est susceptible de redéfinir les propriétés situées de la topicalité, telles qu'elles sont élaborées ensemble par les participants en relation aux contingences du déroulement séquentiel et contextuel de la conversation. Ainsi en est-il par exemple de certaines propriétés identifiées dans la littérature comme définissant le topic: c'est le cas de la propriété "connu" (known) (par opposition à la "nouveauté" censée caractériser le comment ou le rhème, selon les modèles) (cf. p.ex. Chafe, 1994; Givon, 1992; Lambrecht, 1994). Mais alors que la littérature a traité cette propriété de façon ou formelle ou cognitive, il est possible de la traiter comme une caractéristique rendue pertinente et établie par les participants dans l'interaction, qui se manifeste dans le travail public d'établissement d'un savoir commun:

Extrait 6 (Mondada \& Zay 1999:405) (radio)

le cafél ce qu'on ne sait pas toujours/ . est d'origine africainel

Extrait 7 (cité par Sacks \& Schegloff 1979)

1 A well I was the only one other than than the uhm tch

2 Fords?, uh Mrs. Holmes Ford? You know

$3 \quad \mathrm{uh}=[$ the cellist?

4 B [oh yes. She's she's the cellist

5 A Yes. Well she and her husband were there.

Comme on le voit dans le premier exemple, la propriété "connu" peut être modulée de différentes façons, dans le marquage explicite du statut du topic: ici l'incise permet de spécifier le caractère "pas toujours connu" d'une propriété du topic, le rendant ainsi digne d'être mentionné et développé. Dans le second cas, la mention de la référence personnelle, sous forme de nom propre, est énoncée accompagnée par des try markers, des hésitations, des contours interrogatifs, des reprises du nom propre, une 
mention de la catégorie professionnelle de la personne. C'est ainsi que A établit d'abord le statut "connu" (cf. "you know" 2) du topic, avant de continuer à le traiter (par une reprise anaphorique sous forme pronominale).

Par ailleurs, notamment lorsque ce statut n'est pas assuré, les locuteurs peuvent proposer des explicitations du topic, dans des formulations, au sens technique que Heritage \& Watson (1979) donnent à ce terme en développant une idée de Garfinkel \& Sacks (1970) les définissant comme des gloses par lesquelles les locuteurs décrivent à toutes fins pratiques la conversation telle qu'elle est en train de se dérouler.

Ainsi par exemple la formulation du topic peut être une procédure utile pour l'introduire explicitement:

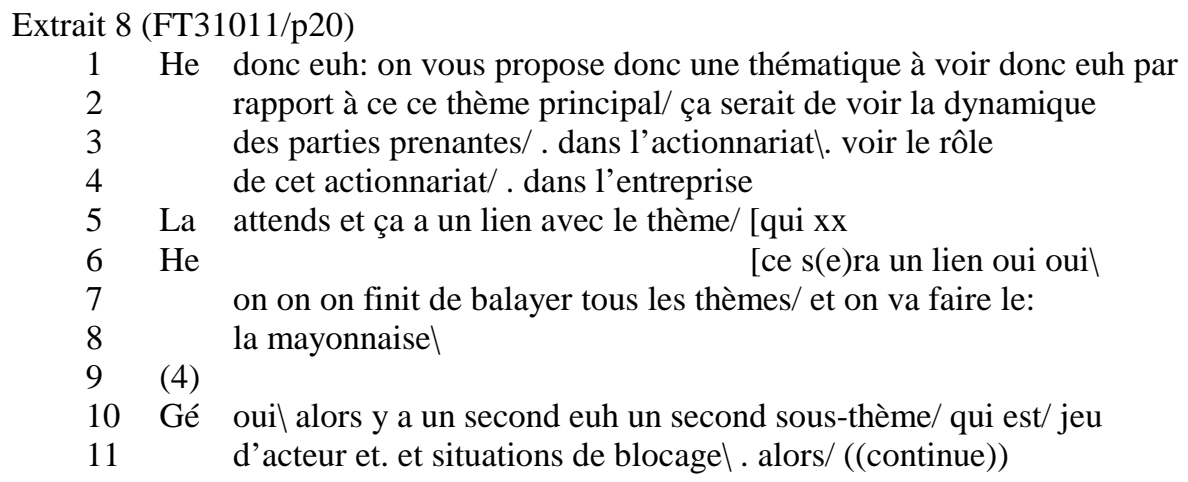

Dans cet extrait, plusieurs propriétés du topic sont explicitement énumérées par les participants: les topics sont hiérarchisés en principal et subordonné (allant de pair avec une éventuelle différenciation terminologique entre "thématique" et "thème"); ils sont aussi linéarisés, pouvant faire l'objet d'énumérations (10); en outre ils obéissent à des contraintes de continuité (5) sans lesquelles leur développement ne peut se faire, voire à des contraintes de cohérence (vernaculairement catégorisée ici en termes de "mayonnaise" 8). Un tel extrait montre combien les locuteurs peuvent, si le contexte le permet ou le demande, expliciter un modèle situé du topic - énoncé non pas de façon générale mais à toutes fins pratiques par rapport à l'activité en cours.

\subsection{Production du topic et appartenance catégorielle des participants}

Dans un exemple célèbre longuement traité par Sacks, un locuteur formule le topic à l'adresse d'un nouvel arrivant qui vient de se joindre à la conversation.

Extrait 9 (Sacks, Lecture 3, fall 1965; 1992, I, 144)

1 Joe we were in an automobile discussion

2 Henry discussing the psychological motives for

3 Mel drag racing on the streets

Joe introduit le topic pour un nouvel arrivant, qui vient d'être présenté au groupe et qui est le destinataire de 1-3. En rendant disponible le topic sur lequel portait la 
discussion en cours, les trois locuteurs invitent le quatrième à y participer (Sacks, 1992, I, 324).

L'activité déployée par Joe, Henry et Mel dans l'exemple précédent ne concerne toutefois pas uniquement le partage d'informations permettant au nouveau venu de participer à la discussion: leur activité, dans la forme qu'elle prend, exhibe une articulation à toutes fins pratiques entre formulation du topic, production d'une forme syntaxique et manifestation de l'appartenance catégorielle des locuteurs (Mondada, 1995b). En effet, les trois locuteurs sont en train de montrer au nouvel arrivé qu'ils constituent un groupe: d'une part, ils le font en proposant un topic qui renvoie à une activité (faire des rodéos avec des voitures) liée à une catégorie d'appartenance particulière (les adolescents qui se livrent à ce genre de loisir), qui permet donc de caractériser les participants (Sacks, 1992, I, 301). D'autre part, ils le font en construisant interactionnellement une forme syntaxique particulière: les trois locuteurs collaborent ensemble pour produire un seul énoncé, qui est ébauché par Joe, relancé par Henry et conclu par Mel. Ils se constituent en tant que groupe en montrant qu'ils font quelque chose ensemble, et plus particulièrement en divisant une tâche, en l'occurrence syntaxique, qui n'est normalement pas facilement divisible (l'énoncé étant une entité caractérisée prototypiquement par son unité et par le fait qu'elle relève généralement d'un seul locuteur) - ceci étant très différent de s'organiser pour faire ensemble une tâche qu'un seul ne pourrait faire. De cette façon, ils montrent qu'ils sont d'accord sur le topic en cours: construire collectivement un énoncé thématique est une activité qui ne peut se réduire à la manifestation d'un accord par le deuxième locuteur à la formulation du topic par le premier locuteur. L'accord thématique est ici rendu visible et exhibé à travers le type d'organisation conversationnelle de la syntaxe.

La définition du topic peut ainsi contribuer au dessin d'un espace de participation où certains interlocuteurs sont désignés comme y appartenant et d'autres sont au contraire exclus: y interviennent des procédés faisant collaborer un proche à l'élaboration du topic, par exemple en déclarant ne plus se souvenir des détails pour lui passer la parole (Goodwin, 1987), ou au contraire des refus de son offre de collaboration, ces deux cas étant illustrés par les extraits ci-dessous:

\section{Extrait $10(\mathrm{tc} 11127 \mathrm{v} / \mathrm{str} / \mathrm{st})$}

$\begin{array}{lll}1 & \text { E } & \text { en cours de: euh de manipulation/ j'ai eu beaucoup de mal à } \\ 2 & & \text { TRAverser la paroi du kyste/ parce qu'elle était extrêmement } \\ 3 & & \begin{array}{l}\text { dure/ . c'est tout ce qui s'est passél j'ai mis un: c-un } \\ \text { drain de gros calibre/ un } 12 \mathrm{~F}=\end{array} \\ 4 & & \text { a }\end{array}$

\section{Extrait 11 (P33/fgc21044e)}

1 I quand ils vont monter i- ils ils tombent

2 A et il met des clous par terre

3 I pis il emmène le: ... à repasser là:

4 A [le fer ouais

5 I [le fer à repasser en haut/ et pis y a un voleur qui entre

6 dans la cave/ 


$\begin{array}{clll} & 7 & \text { A } & \text { il voulait allu[mer } \\ 8 & \text { I } & \text { [pis il voulait allumer la lumière (rires) } \\ & 9 & & \text { et pis il a tiré le filet y le . le } \\ -> & 10 & \text { F } & \text { le feu } \\ -> & 11 & \text { I } & \text { no:n y a le } \\ 12 & \text { A } & \text { le fer . [à repasser } \\ 13 & \text { X } & \text { [à repasser } \\ 14 & \text { I } & \text { le fer à repasser qui va ici/ après i se brûle }\end{array}$

Dans l'extrait $10 \mathrm{~L}$ continue par une relative la description ébauchée par E: ce faisant il exhibe le fait qu'il appartient à la même équipe médicale et que ce qui est présenté est une version collective du geste chirurgical qui a été effectué; dans l'extrait 11 , nous avons aussi à faire à un groupe, composé de $\mathrm{A}$ et I, qui est engagé dans une activité collaborative de compte-rendu d'un film: leur appartenance au même groupe est accomplie par une distribution du travail de formulation, l'un prenant la relève de l'autre pour la faire progresser. Ce travail de constitution de A et I comme appartenant à la catégorie des "narrateurs", opposée à celle du "public" est effectué dans la gestion de l'activité discursive; lorsque F (10) tente d'y collaborer son apport est refusé et par là son inscription dans le même espace de participation.

Teresaki $(1976,23)$ propose dans ce sens que la gestion du topic fait intervenir des catégories variées, non seulement le couple "recipient"/“deliver" mais aussi le couple "already informed"/"to be informed", qui définissent des droits et obligations à la parole particuliers et des possibilités de collaboration (ainsi il peut y avoir plusieurs locuteurs catégorisables comme "already informed" qui peuvent se partager ou se confier mutuellement le travail d'annoncer un topic ou une nouvelle).

La gestion des topics dans la conversation est donc doublement liée aux procédures de catégorisation des locuteurs: d'une part, en gérant le topic d'une certaine manière, les locuteurs déploient leurs appartenances catégorielles, voire les instaurent par leur façon de faire; d'autre part, l'identification catégorielle des participants rend disponible un certain nombre de topics qui pourront alimenter la conversation, même si les interlocuteurs ne se connaissent pas, du seul fait qu'ils sont générés par des connaissances typiques liées à la catégorie (Sacks, 1972). Ainsi un Français arrivant aux Etats-Unis se verra interrogé sur ce qu'il pense de la nourriture américaine, même si son interlocuteur ignore s'il est un gourmet ou quels sont ses goûts personnels, par le seul appariement typique entre l'appartenance à une catégorie nationale et une certaine culture gastronomique. Ainsi, au contraire, certains topics sont à éviter avec certaines catégories de personnes, comme le dit bien le proverbe "On ne parle pas de corde dans la maison d'un pendu".

\subsection{Articuler des positions séquentielles, des procédures, des configurations thématiques et des activités de marquage des participants}

Un argument, un fait, un événement n'est jamais en soi et a priori un topic - il faut le configurer pour cela, il faut en faire un talk-aboutable (Sacks, 1992, II, 568). Le statut de topic s'accomplit dans et par l'interaction elle-même: cela dépend d'une part du locuteur qui l'introduit, qui pour cela doit transformer "un topic dont j'ai envie de 
parler" en "un topic adéquat pour telle ou telle occasion conversationnelle particulière", qui puisse être inséré dans la conversation, qui puisse intéresser les partenaires, qui déclenche leur collaboration pour le développer. Cela dépend aussi de ce que les participants vont en faire, de la trajectoire qu'ils vont lui offrir.

Ce problème pratique concerne quatre aspects des processus thématiques qui sont liés mais que l'on peut distinguer analytiquement:

- D'une part il concerne un ensemble de positions séquentielles, qui n'offrent pas les mêmes opportunités pour le placement du topic: il y a ainsi des positions sensibles dans l'interaction, comme le début ou la fin, où il est attendu que des introductions thématiques aient lieu; il y a aussi des positions qui apparaissent occasionnellement au fil de la conversation, qui ne sont pas prévisibles ou programmables en tant que telles. Ces aspects ont bien été traités par Button \& Casey $(1984,1985)$ et nous n'allons pas y revenir ici.

- D'autre part il y a des procédures qui provoquent l'opportunité d'introduire un topic disjoint des précédents, d'autres qui exploitent des opportunités pour s'y rattacher; il y a des procédures de premier locuteur et des procédures de deuxième locuteur, qui exploitent favorablement des enchaînements permettant d'introduire des éléments en les accrochant à un argument soulevé par un autre (cf. infra 5.)

- Ces procédures produisent différentes configurations thématiques: configurations délimitées, clôturées, bien distinctes de celles qui les précèdent et qui les suivent, ou configurations ouvertes, présentant des espaces de transition avec d'autres configurations (cf. infra 4.)

- Enfin, ces configurations sont produites de façon reconnaissable pour les interlocuteurs grâce à l'exploitation située de ressources linguistiques permettant de marquer la construction topicale en train de se faire (cf. infra 6.)

Ces dimensions sont liées: certaines procédures organisent préférentiellement certains types de configuration, et occupent certaines positions, qui prennent une valeur particulière par rapport à l'organisation de la conversation et qui recourent à des matériaux linguistiques spécifiques pour produire leur identifiabilité.

\section{CONFIGURATIONS THÉMATIQUES ET PROCÉDURES DE GESTION DU TOPIC}

Par leur façon de gérer le topic, les participants l'informent comme appartenant à une configuration ou à une autre. Ce n'est donc pas le topic en soi qui possède une configuration particulière, mais l'activité thématique des locuteurs qui le structure de telle ou telle façon en s'ajustant à lui comme relevant de telle ou telle configuration. Nous aborderons successivement deux grands types de configurations: celles qui caractérisent des topics planifiés par opposition à des topics improvisés (4.1.), des topics se transformant progressivement en d'autres topics par opposition à des topics dotés de frontières nettes (4.2.).

\subsection{Topics planifiés et topics improvisés}


Contrairement à la conversation ordinaire spontanée, certains types d'interactions sont organisés par un agenda de topics prévus à l'avance. Tel est le cas de réunions où un ordre du jour est énoncé par le président de séance et souvent distribué sous forme écrite aux participants.

Même dans ce cas, l'ordre du jour ne fonctionne pas comme une simple détermination de l'interaction à venir: il est en effet intéressant d'observer la façon dont le cours des événements est ajusté de façon locale à l'ordre du jour et dont l'ordre du jour est pratiquement interprété, avec tous les ajustements ad hoc que comporte l'accomplissement indexical de cet ordre. Dans de tels cas, il y a donc moins une relation de détermination univoque qu'une relation réflexive qui s'établit entre les deux: en s'adaptant à l'ordre du jour, l'événement contribue à sa lecture et donc à sa (re)structuration. L'ordre du jour soulève ainsi les mêmes problèmes que d'autres instructions pour l'action, comme par exemple les manuels d'utilisation ou les recettes de cuisine, voire de façon générale les invitations à suivre une règle: l'indexicalité des activités rend impossible une détermination littérale de la part de la description de l'action postulée, et pemet à l'analyste de poser la question de la façon dont l'adéquation de l'action à l'instruction est bricolée in situ par les participants.

Reste que les acteurs qui recourent à des agendas thématiques le font dans le but de structurer et de contraindre le déroulement de l'action, éventuellement face à des interlocuteurs qui non seulement négocieront l'interprétation de l'agenda mais encore le subvertiront en ayant d'autres programmes thématiques. On peut ainsi observer la façon dont la présentation d'un agenda thématique prétend structurer la suite de l'interaction: l'annonce de trois points thématiques à développer, par exemple, a pour effet de suspendre éventuellement l'alternance des tours de parole jusqu'au moment où les trois points ont été traités - comme l'annonce d'un récit ou du début d'une blague suspend jusqu'à sa fin les interventions des autres participants (Jefferson, 1978). De même, le fait de définir de manière très explicite le topic que l'on veut traiter ou qui est en train d'être traité constitue une façon d'imposer une sorte de titre à une séquence, et de contraindre ainsi la suite de son développement, qui ne pourra pas ne pas en traiter. Ces procédures montrent que même dans la conversation ordinaire, ou dans des formes d'interaction informelle, les locuteurs peuvent recourir à des moyens de planification du topic pour contraindre son élaboration.

Toutefois les locuteurs ne se joignent généralement pas à une conversation ordinaire spontanée en ayant un programme thématique défini comme tel; ceci signifie que, tout en ayant à disposition des objets de discours possibles, ils ne les manifestent pas d'emblée et n'imposent généralement pas une liste de topics dont devra traiter la conversation. Les locuteurs s'orientent vers le fait que les talk-aboutables devront être introduits de façon pertinente dans le cours de la conversation: «this is not exactly an "agenda", for it includes relevant "talkables" which a party is not oriented to introducing into the talk but which could "come up" relevantly » (Schegloff, 1986, 116). Sacks fait remarquer que ce type de placement est caractéristique des conversations qui se déroulent normalement: « in [...] a good conversation what you would find is that new topics are never "introduced", they just happen along » (1992, II, 352). Et il fait remarquer qu'un «bon » topic est moins celui qui retient longtemps l'attention des interlocuteurs que celui qui permet des transitions vers d'autres topics sans que soient nécessaires des marqueurs d'introduction spécifiques. En caractérisant 
la conversation par sa dynamique thématique, liée à sa sensibilité au contexte et aux participants, qui la différencie radicalement d'autres interactions prioritairement orientées vers le traitement d'objets de discours précis et objectivés, Simmel fait écho à cette intuition, en affirmant que la capacité de changer de topic facilement et rapidement définit une conversation sociable (cité par Bergmann, 1990, 215).

La conversation se caractériserait donc par l'importance que prend son organisation thématique locale. Bergmann (1990) exploite cette notion dans deux sens différents: d'une part les topics ont une dimension locale dans le sens où ils se configurent dans le déploiement de la séquentialité, tour par tour (cf. supra); d'autre part la dimension locale des topics est constituée par le fait que dans ce type de conversation les interlocuteurs exploitent souvent un événement, un fait, un détail de leur environnement perceptible immédiat pour en faire un topic conversationnel. Cette façon de faire, qui à la fois provoque et rend intelligible une grande hétérogénéité et discontinuité thématiques, est typique des conversations durant les repas, où les participants peuvent orienter leur attention thématique vers les plats qu'ils sont en train de manger, vers le chien qui quémande un reste ou vers un bruit inhabituel dans la rue (Erickson, 1981). Tout élément de l'environnement est ainsi susceptible de devenir un topic et d'être introduit abruptement du seul fait de sa disponibilité perceptive: là encore ce n'est pas uniquement la saillance de l'objet dans l'environnement qui déclenche son élaboration thématique, mais son émergence dans le champ de l'attention collective des participants, par leur activité discursive menée collaborativement.

Ce réservoir local de topics peut devenir une ressource utile pour redonner vie à une conversation; il peut aussi offrir des alternatives utiles lorsque la discussion aborde des topics embarassants ou conflictuels, en permettant aux participants de changer rapidement de sujet (Bergmann, 1990, 211, 214).

Ce type de ressource n'est pas ou peu disponible pour des interactions formelles, où au contraire tout est fait pour que les topics traités soient ceux qui ont été prévus et non ceux qui émergent de l'interaction et où on pourrait dire que le contrôle rituel exercé sur l'environnement, souvent soigneusement préparé et mis en scène pour la rencontre, vise précisément à éviter que des objets extérieurs au dispositif de la rencontre puissent y faire irruption.

\subsection{Topics à développement progressif et topics avec frontière}

La fluidité et la richesse thématique de la conversation se caractérise ultérieurement par le fait que ses locuteurs passent d'un topic à un autre par transitions progressives, sans que l'on puisse dire où exactement on a cessé de parler d'un topic et où on a commencé à en aborder un autre. Ceci serait l'indice d'une bonne conversation, c'est-à-dire d'une conversation qui avance d'une façon bien huilée, fluide, par opposition à une conversation dont les topics s'épuiseraient les uns après les autres, en portant à la clôture de la conversation elle-même, ou qui avancerait à coup de ruptures, d'abandons de topics embarassants et de nouvelles introductions (Sacks, 1992, II, 566).

Contrairement aux topics à développement progressif, les topics avec frontière sont clairement délimités, par des introductions explicites d'une part (cf. Button \& Casey, 1984), par des clôtures tout aussi nettes d'autre part. Ces configurations thématiques conviennent mieux aux interactions formelles, où les locuteurs considèrent 
important de traiter exhaustivement un topic et de ne pas passer au suivant avant de l'avoir épuisé. Dans la conversation spontanée aussi, des techniques sont disponibles pour marquer et provoquer la fin observable d'un topic: ainsi le destinataire du développement thématique peut provoquer l'abandon et la clôture d'un topic par des procédures qui consistent d'abord à énoncer des commentaires interactionnellement alignés avec ce qui est en train de se dire, et de les faire suivre ensuite par un mouvement thématique radicalement disjoint, permettant le passage à un autre topic (Jefferson, 1993).

Les transitions progressives sont organisées de différentes façons et donnent lieu à des procédures variées de gestion et d'introduction. De façon générale elles préfèrent des configurations thématiques ouvertes, dont la caractéristique est moins d'être des topics foisonnants, susceptibles de longs développements, que d'être des topics qui permettent de passer facilement à d'autres (Sacks, 1992, II, 352). Ces deux dernières possibilités engendrent des façons de parler différemment: on peut distinguer entre "parler d'un topic" (talking about a topic) et "parler topicalement" (talking topically) (Sacks, 1992, I, 762). Les locuteurs font des choix pouvant aller dans un sens ou dans l'autre: ils peuvent vouloir maintenir l'attention sur un topic et en exploiter différentes facettes; ils peuvent aussi provoquer des transitions vers d'autres topics voisins. Des visées différentes - stabiliser un topic ou le faire dériver - déclenchent des choix de configurations et de formulations différentes: ainsi selon Sacks (1992, I, 758) le locuteur voulant maintenir la conversation sur le topic d'un week-end sportif passé à surfer choisira une formulation du type "J'ai été à Malibu Beach ce week-end", centrée sur un toponyme renvoyant à un lieu réputé où on pratique le surf, plutôt que "J'ai été faire du surf ce week-end", centrée sur l'activité: la première invite à développer le topic du surf, alors que la deuxième peut susciter des enchaînements du type "Et moi j'ai été faire du ski", qui déclinent plutôt les activités que l'on peut faire le week-end.

Dans ce sens, les mouvements progressifs peuvent réaliser aussi bien une continuité qu'une discontinuité thématique. Ils peuvent par exemple connecter deux topics autonomes en introduisant un topic qui leur est commun et qui peut fonctionner comme une passerelle de l'un à l'autre (Sacks, 1992, II, 300). Ils peuvent aussi générer des sous-topics qui continuent à être traités comme relevant du topic principal. Un mouvement progressif peut ainsi lier des topics reliés comme des topics disjoints - leurs relations ne dépendant d'ailleurs pas de relations sémantiques abstraites mais de mises en relations effectuées par les locuteurs eux-mêmes, de façon contextuelle et à toutes fins pratiques.

Un élément charnière dans un tour de parole peut être susceptible de plusieurs développements thématiques possibles et donner effectivement lieu à des enchaînements différents. Ainsi deux locuteurs peuvent proposer deux développements distincts et se trouver dans une situation de concurrence thématique. Une façon pour les participants de gérer cette situation sans pour autant se rallier à un seul topic consiste à maintenir chacun sa ligne thématique, en se référant dans sa prise de parole, non pas au tour immédiatement précédent de l'autre locuteur, mais à son propre tour, comme si l'on ignorait la contribution de l'autre: Sacks appelle cette technique skip-connecting (1992, II, 349). Ce qui caractérise ce type de développement est le fait que les deux topics en compétition ne sont pas des topics radicalement différents, mais des topics qui ont une origine commune et qui sont souvent assez proches, tout en représentant deux 
lignes thématiques distinctes. C'est pour cela que les deux trajectoires caractérisant le skip-connecting peuvent converger à un moment donné et collaborer ensemble au lieu de se concurrencer.

Le développement progressif du topic permet aussi de distinguer entre stratégies du premier et du second locuteur: alors que les stratégies du premier locuteur sont introductives, initiatives et tendent à exercer un effet de contrainte prospective sur la suite, les stratégies de second locuteur consistent à exploiter un enchaînement, à s'appuyer sur la contribution du premier pour insérer du matériau thématique inédit. Le second locuteur exploite des éléments du tour précédent pour construire et imposer des relations de similarité et de cohérence avec eux (Sacks, 1992, II, 255). Un exemple en est donné dans l'échange suivant:

\section{Extrait 12 (FR/inc)}

1 A mais je crois/ dans un endroit qui est particulièrement:

2 intéressant/ et: . je crois qu'il soulève un problème de

3 fond/ c'est le problème du FEU/ l'été/ . et je crois qu'il

4 faut le soulever ce problème parce que. on est jamais

5 assez préventif/ et on: . et quand on va voir cette ce film

6 que nous avons: [tourné sur la montagne SAINte-Victoire/

7 B $\quad[x x x x x x$

8 B ah oui à Aix-[en-Provence/

9 A [c'est un endroit que j'adore/ vraiment qui

10 est merveilleux/

11 B qui a été défiguré COMplè[tement।

12 A [qui a été DEfiguré complètement)

13 alors il reste encore quelques parties côté Vauvenargues et::

14 B oui oui

15 A et je crois que ça va revivre/ heureusement/ grâce à

16 l'effort de:

17 B Cézanne a dû se retourner dans sa tombel

18 C mais qu'il risque de l'être encore plus [par le: TGV/ et: xxx

19 A [NON C'est ça/ non

20 non c'est ça/ y a le problème du TGV qui: qui vient se

21 greffer là-dessus/

$22 \mathrm{C} \quad \mathrm{xx}[\mathrm{x}$ xxxx

23 A [faut faire très attention/ je crois que c'est notre

24 euh: c'est notre environnement/ c'est notre vie/ c'est

25 notre SOUffle de vie qu'est en: . qu'est en cause/ il

26 faut: sauvegarder l'ensemble de la nature/ hein/

$\mathrm{B}$ en 11 complète l'énoncé de $\mathrm{A}$, en y ajoutant une relative: ce mouvement opère doublement, d'une part en produisant collaborativement un énoncé et d'autre part en développant un argument opposé à celui de A (celui-ci insiste sur les beautés à préserver de la Sainte-Victoire, alors que B souligne les destructions massives qui l'affectent). A reprend la relative de B en 12, tout en reprenant aussi sa propre ligne de développement; de son côté, $\mathrm{B}$ intervient à nouveau en 17 , en établissant moins un 
rapport avec le tour qui précède immédiatement qu'avec son propre tour en 11. Donc soit A soit B enchaînent thématiquement sur leurs propres conkibutionss en ignorant celle immédiatement précédente de leur interlocuteur, par la technique du skipconnecting. La résolution de cet enchaînement concurrentiel a lieu, ainsi que Sacks le décrit, au moyen de l'intervention d'un tiers (1992, II, 351), en l'occurrence C en 18, qui, en intervenant après $\mathrm{B}$, fournit un développement qui n'est pas compétitif mais additif au dernier tour et, de cette façon, sanctionne positivement la ligne thématique de B.

Si l'on compare les «méthodes » de gestion du topic de A et de B, on remarque que A recourt à des formulations qui lui permettent d'énoncer le topic de façon particulièrement explicite, en termes de "problème": c'est le cas en 2-4 ("le problème du feu", avec 3 reprises, qui opèrent son introduction, spécification, extraction); c'est le cas aussi en 20, lorsque $\mathrm{A}$ intègre la ligne thématique de $\mathrm{B}$ par la même mise en forme ("le problème du TGV"). Cette technique de A est une façon non seulement de mettre en évidence le topic, mais d'en assurer la projection sur plusieurs séquences successives (la technique de A semble fonctionner de façon à lier l'importance des moyens d'introduction du topic et l'importance que celui-ci aura sur la suite de la conversation): c'est donc une technique qui se fonde sur le marquage comme préface d'un long développement et par conséquent sur le maintien de la parole par le locuteur. Par contre, la technique de $\mathrm{B}$ utilise des ressources qui relèvent moins du marquage que du positionnement séquentiel: sa façon de rendre opérante une ligne thématique consiste à exploiter les enchaînements séquentiels possibles ou à en proposer d'alternatifs, en comptant sur la dynamique du passage du tour de parole, et notamment sur d'autres interventions que la sienne.

On voit donc d'une part que les méthodes de gestion, de contrôle, de projection du topic peuvent relever autant de "techniques de premier locuteur", projetant une suite dont les locuteurs successifs vont devoir tenir compte, que de "techniques de second locuteur", qui réinterprètent ce qui précède pour le transformer. D'autre part, on voit aussi que même lorsque les développements topicaux divergent, les locuteurs sont attentifs aux apports de leurs interlocuteurs pour co-élaborer leur ligne concurrentielle.

\section{PROCÉDÉS COLLABORATIFS : LA CO-CONSTRUCTION DES TOPICS}

Un objet de discours n'appartient en effet pas en propre à un seul locuteur, mais, une fois introduit, il est pris en charge par tous les participants (Mondada, 1995b). Ceux-ci interviennent dans son développement et dans sa configuration de différentes façons: même lorsqu'ils se limitent à en accompagner la formulation par des signes d'acquiescement, voire des évaluations et des commentaires, ils interviennent d'une certaine manière sur sa forme, que l'énonciateur ajuste réflexivement à eux. En outre, ils peuvent intervenir de façon plus radicale, en collaborant à l'établissement même de ses contours.

Tel est le cas de l'extrait suivant:

Extrait 13 (FT31011) (réunion de travail)

1 TRO l'o- l'objectif c'est quoi alors/ en fait \du thème/ . 


\begin{tabular}{|c|c|c|}
\hline 2 & & que vous proposezl \\
\hline 3 & (2) & \\
\hline & PRE & c'est-à-dire/ est est-ce qu'il existe une prise de \\
\hline & & pouvoir par contamination/ hein au travers \\
\hline & & de [de quand on parle de rapprochement d'entreprise/] \\
\hline & TRO & [c'est l'évolution des modèles cul]turels ou \\
\hline & & est-ce que c'est euh:: 1- \\
\hline & PA & qui va bouffer l'autrel \\
\hline 10 & TRO & ouais \} $\\
{\hline 11} &{\text { PRE }} &{\text { non c'est [l'évolution/ }} \\
{\hline 12} &{\text { PA }} &{\text { [d'Orange ou de France Télécom }} \\
{\hline 13} &{\text { B }} &{\text { par exemple }} \\
{\hline 14} &{\text { PRE }} &{\text { c'est pas [c'est pas pour bouffer/ }} \\
{\hline 15} &{\text { TRO }} &{\text { [est-ce que c'est }} \\
{\hline 16} &{\text { PA }} &{\text { [xx finalement comme ça/ }} \\
{\hline 17} &{\mathrm{C}} &{\text { [non c'est pas bouffer/ c'est l'évolution du du- }} \\
{\hline 18} &{\text { PA }} &{\text { attends }} \\
{\hline 19} &{\text { PRE }} &{\text { y a y a y aly a Orange qui est le petit/ . et qui euh }} \\
{\hline 20} &{ } &{\text { et et qui euh euh et vraiment un contrat/ c'est France }} \\
{\hline 21} &{ } &{\text { Télécom qui dit } \mathrm{OK} \backslash \text { vous vous êtes comme } \mathrm{CA} / \text { nous on }} \\
{\hline 22} &{ } &{\text { on on a tel profil/ . euhl. moi ce qui m'intéresse/ }} \\
{\hline} &{ } &{\text { c'est c'est votre nom et votre philosophie pa- parce }} \\
{\hline} &{ } &{\text { que j'y crois et en plus je veux qu'on la contaminel }} \\
$\hline
\end{tabular}

Encore une fois, les participants exhibent leur orientation vers non seulement ce qu'est le topic (1. 1) mais encore vers sa formulation, ses contours, ses caractéristiques sémantiques. Cela déclenche une reformulation du topic (4) effectuée par Présenti (46) mais immédiatement concurrencée par Trovin en chevauchement (7-8). La version de Présenti est donc confrontée à une double version alternative de Trovin, la seconde n'étant que partiellement énoncée (“ou est-ce que c'est euh:: 1-" 7-8) et complétée par Pasquin (9). L'alternative est donc collaborativement formulée par Trovin et Pasquin (qui continue ligne 12), ratifiée par Trovin (10), niée par Présenti (11) qui esquisse une double reformulation de la sienne (positive 11 et négative 14). La seconde nie celle de Trovin et Pasquin et est reprise par un autre participant, Carven (17), dans un passage où les chevauchements sont multiples. Ce n'est qu'après une régulation explicite du mode de l'échange de la part de Pasquin (18) que Présenti pourra reformuler sa version (19-24). On voit que les interventions des autres participants ne laissent pas intacte la formulation de Présenti, qui subit un ensemble de transformations de la première (4-6) à la dernière (19-24) version: la première est plus abstraite, caractérisée par des nominalisations d'actions, alors que la seconde est ancrée dans les actions d'un acteur particulier mis en scène à la première personne.

On peut ainsi dire qu'en cas de désaccord comme en cas d'accord et de construction collaborative, les objets de discours sont collectivement élaborés par les participants. Cette co-élaboration est notamment rendue possible grâce à la façon dont les participants mobilisent les ressources grammaticales dans l'interaction en train de se faire. 
En particulier, une façon pour les participants de montrer qu'ils sont en train coélaborer l'objet de discours consiste à construire collaborativement des énoncés, comme dans les extraits suivants:

\begin{tabular}{|c|c|c|}
\hline \multicolumn{3}{|c|}{ Extrait 14 (une95boe) } \\
\hline 1 & $\mathrm{C}$ & enfin/ y a une année maintenant qu'il a deux qu'il a deux \\
\hline 2 & & passeports $\backslash$ \\
\hline 3 & B & ah il en a deux/ \\
\hline 4 & $\mathrm{C}$ & mais à dix-huit ans ils lui ont dit euh: \\
\hline 5 & B & qu'il devait choisir/ \\
\hline 6 & $\mathrm{C}$ & qu'il devait choisir $\backslash$ puis lui il avait cru que il a- il \\
\hline 7 & & avait REnié l'italienne/ \\
\hline
\end{tabular}

Extrait 15 (une95boe)

1 C il croyait aussi qu'en signant/ parc'qu'j'sais plus/ il se

2 souvenait qu'à dix-huit ans il avait dû signer quelque

$3 \quad$ cho(se)\ .. il avait l'impression qu'il avait: euh dû: ouais

$4 \quad$ [xx

5 B $\quad[<$ renoncer $(($ accéléré $))>$

6 C renoncer

7 B renoncer à sa: [à sa nationalité italienne

8 C [pis en fait pas du tout।

H. Sacks a montré dans ses cours dès la fin des années 60 que ces énoncés collaboratifs (joint productions) étaient une façon à la fois de faire quelque chose ensemble - quelque chose que la littérature considère habituellement comme relevant de la compétence syntaxique d'un seul locuteur - et d'exhiber ainsi les participants comme formant un groupe (Sacks, 1992, I, 321ss; Lerner, 1991; cf. Mondada, 1999 pour d'autres références et analyses). Ces procédés de composition collective d'énoncés montrent combien la grammaire peut être exploitée de façon située pour des actions collaboratives. C'est dans ce cadre qu'une reformulation de la façon de considérer les marquages grammaticaux des objets de discours est possible.

\section{RESSOURCES GRAMMATICALES ET ORGANISATION SÉQUENTIELLE}

La littérature sur les processus de référenciation, de thématisation et d'anaphorisation a buté contre la polyfonctionnalité des marqueurs grammaticaux des objets de discours: en effet ceux-ci ne sont pas biunivoquement articulés à une fonction discursive précise - le pronom ne marquant pas toujours une information ancienne ou connue, la dislocation à gauche étant parfois exploitée pour introduire un nouvel objet, parfois pour en mettre un relief un ancien, etc. (cf. Apothéloz, 1997; Cornish, 1999, Jubran, Urbano \& Koch, 1992). Ces constats, dont la littérature abonde, montrent la difficulté d'établir une articulation générale entre formes et fonctions, fondée implicitement sur une conception selon laquelle la langue "coderait" des statuts des 
référents ou des informations. Une approche alternative se penche plutôt sur les activités par lesquelles les locuteurs rendent localement et publiquement intelligibles leur choix expressifs, en ne se limitant pas simplement à "coder" des entités référentielles ou cognitives, mais en bricolant contextuellement les marquages les plus appropriés à la situation communicative. Ainsi la valeur des marqueurs utilisés est moins à rapporter à un modèle général de la fonctionnalité des ressources linguistiques et davantage à la façon dont elles sont utilisées de façon située et dont elles acquièrent un sens rendu manifeste par leur positionnement séquentiel particulier, lié à l'organisation de l'interaction. L'analyse se concentre alors sur les procédés par lesquels les participants structurent leurs activités en utilisant de façon située certaines formes linguistiques.

L'extrait suivant permet de caractériser cette approche:

Extrait 16 (HR20118/MUL/ap2-1157-1181)

1 W müssten wir schon nochmal genauer defi[nieren\& on devrait encore définir une fois plus précisément\&

$2 \mathrm{~V} \quad[\mathrm{mhm}=\mathrm{mhm}$

3 W \&was wir unter äh fondation wirklich verstehen $\backslash$ äh::. weil \&ce qu'on comprend vraiment comme étant la fondation $\backslash$ äh:: .

$4 \quad$ es eben doch verwandte begriffe auch gibtl. und und parce qu'il y a justement des concepts apparentés\. et et

5 und phänomene et des phénomènes

$6 \quad(2 s)$

7 W ich mein der koriolan ist kein- deswegen weil er j'veux dire Coriolan est pour ça n'est pas parce qu'il

8 verurteilt wird ist [er noch kein fondatEUR/ est jugé [il n'est pas encore un fondatEUR/

$9 \mathrm{~V}$

$[\mathrm{mhm}=\mathrm{mhm}$

$10 \mathrm{~W} \quad[<$ ebenfalls $\backslash$. das $\mathrm{xxxx}(($ bas $))>$

$[<$ pareillement .. le $x x x x$

$11 \mathrm{~V}$ [le: le: le: jugement/ .. le jugement n'est pas fondateur

$12(4 \mathrm{~s})$

$13 \mathrm{~W}$ also er er grÜndet nichts\. so[ndern er ist allenfalls donc il il ne fonde rien]. ma[is il est de toute façon

$15 \mathrm{~W}$ so ein passiv. eine passive rolle dabeil un passif. il a un rôle passif

$16 \mathrm{~V}$ NON\ lui-même depuis lui-même il est victime/

$17 \mathrm{~W} \quad$ [ (er ist das opfer) $\mathrm{ja}=\mathrm{ja} \backslash \mathrm{ja}=\mathrm{jal}]$ [ (il est la victime) oui $=$ oui $\backslash$ oui $=$ oui $]$

$18 \mathrm{~V}$ [mais sa figure est fondatrice/. c'est sa fIgUre qui est

19 fondatricel]

20 W ja=JA: aber oui=OUI: mais

$21 \mathrm{~V}$ d'une procédurel. d'un événement/. d'une procédurel. et 

et et susceptible de reproduction $\backslash=$

$23 \mathrm{~W}=(\mathrm{ja})$ aber ist das wirklich fondatrice

24 (8s) $=($ oui) mais est-ce que c'est vraiement fondatrice

$25 \mathrm{~V}$ <euh:.: euh:: oui/ moi je. je pense oui/ enfin $\backslash($ (bas $))>$

Dans cet extrait d'une réunion de travail bilingue, deux professeurs, l'un suisse, $\mathrm{W}$, et l'autre français, $\mathrm{V}$, sont en train de discuter sur un projet de recherche scientifique qu'ils développent ensemble, portant notamment sur la notion de "fonction fondatrice", qui est ainsi un objet de discours qui accompagne leur travail sur plusieurs années, qui est donc aussi un "objet de savoir" (Mondada, 2000b). Leur discussion est un exemple intéressant d'élaboration collective du topic dans un mixte d'accord et de désaccord. A propos de Coriolan, un héros romain sur lequel ils travaillent, ils sont d'accord pour considérer la non pertinence du jugement pour la fondation $(8,11)$, sur le fait que Coriolan ne fonde rien $(13,14)$, sur le rôle de victime de Coriolan $(16,17)$. Toutefois, au-delà de cet accord, une divergence apparaît qui aboutit à un désaccord (23-25): elle concerne précisément la façon dont l'objet de discours est conçu, dont la continuité topicale est manifestée et construite par les deux interlocuteurs. Alors que W se réfère de façon constante à Coriolan, par le pronom "er" ("il"), en faisant ainsi de la discussion une interaction topicalement centrée sur ce personnage historique, au contraire V mentionne des objets plus abstraits (V 18 "la figure" vs W "il", V 11 "le jugement" $v s \mathrm{~W}$ "il est jugé") et change d'objet à plusieurs reprises, qu'il marque de façon à les mettre en relief et à souligner un contraste possible (par une construction disloquée, 1 . 11, par une construction clivée 1.18 , et en faisant référence une seule fois à Coriolan, par un pronom tonique disloqué "lui-même il"). On peut constater ainsi que V et $\mathrm{W}$ utilisent des ressources formelles très différentes pour marquer des objets de discours ainsi rendus divergents: alors que $\mathrm{W}$ montre qu'il considère comme allant de soi le maintien topical de Coriolan, $\mathrm{V}$ montre au contraire que l'identification du topic pertinent pose problème. C'est dans le contraste entre les deux, en même temps que dans leur développement séquentiellement imbriqué, que leurs choix grammaticaux prennent leur sens. Ainsi, par exemple, la dislocation à gauche peut intervenir dans un contexte où il $\mathrm{y}$ a désaccord et permettre une organisation séquentielle qui retarde l'apparition de ce désaccord (de Fornel, 1988).

\section{EN GUISE DE CONCLUSION}

Nous avons insisté dans ce texte sur le caractère dynamique et localement construit des objets de discours dans l'interaction verbale. Cette attention particulière nous invite à analyser moins les contenus sémantiques des objets de discours que les procédés par lesquels les participants élaborent interactivement leurs topics. Cette approche procédurale insiste donc sur les activités langagières des participants, où ceux-ci agissent et interprètent à la fois leur action, en lui fournissant son intelligibilité et son sens au fur et à mesure qu'ils l'organisent collectivement. Les topics sont avant tout définis par les interactants — avant que par l'analyste —; ils ne dépendent jamais d'un seul locuteur qui les contrôlerait et les développerait solitairement, mais du 
collectif des participants qui interviennent dans leur élaboration en les stabilisant, en les renforçant, voire en les réifiant, ou bien en les destabilisant, en les mettant en question, ou en les détournant. Dans ces dynamiques, et pour organiser l'interaction, les participants recourent aux ressources de la grammaire qu'ils utilisent à toutes fins pratiques, en les "bricolant" de façon située, en en exploitant les propriétés typiques mais aussi en faisant émerger d'autres principes d'ordre, de façon locale, contingente et liée à l'action.

C'est ainsi que l'approche des topics repose sur une triple préoccupation analytique, concernant l'organisation séquentielle de l'interaction, la configuration émergente de la grammaire, et les processus de référenciation entendus comme l'élaboration de versions publiques du monde.

\section{Conventions de transcription}

$\begin{array}{ll}{[} & \text { chevauchements } \\ \ldots \ldots \ldots & \text { pauses inférieures à la seconde } \\ (2 \mathrm{~s}) & \text { pauses en secondes } \\ \text { Xxx } & \text { segment inaudible } \\ / 1 & \text { intonation montante/descendante } \\ \text { exTRA } & \text { segment accentué } \\ \vdots & \text { allongement vocalique } \\ ((\text { rire })) & \text { phénomènes non transcrits } \\ <> & \text { délimitation des phénomènes entre }(()) \\ \& & \text { continuation du tour de parole } \\ = & \text { enchaînement rapide } \\ \text { par- } & \text { troncation }\end{array}$

Les extraits sont caractérisés par des codes lorsqu'ils sont tirés de nos propres corpus; ils signalent la référence bibliographique lorsqu'ils sont tirés d'autres auteurs.

Dans le dernier extrait la transcription originale est suivie, ligne par ligne, par une traduction en italique qui est donnée à titre indicatif pour faciliter la lecture de l'original sur lequel est basée l'analyse.

\section{RÉFÉRENCES}

APOTHELOZ, D. (1997), Les dislocations à gauche et à droite dans la construction des schématisations. in D. Miéville, A. Berrendonner, (eds.), Logique, discours et pensée. Mélanges offerts à J.-B. Grize. Bern: Lang, 183-217.

BANGE, P. (1992). Analyse Conversationnelle et Théorie de L'Action. Paris: Hatier/Didier Erudition.

BERGMANN, J. (1990). On the local sensitivity of conversation. In I. Markova \& K. Foppa (Eds.), The Dynamics of Dialogue (pp. 201-226). New York: Harvester Wheatsheaf.

BERTHOUD, A.-C., \& MONDADA, L. (1992). Apprendre à entrer en matière dans l'interaction: Acquisition et co-construction des topics en L2. AILE, 1, 107-142. 
BUTTON, G., \& CASEY, N. (1984). Generating topic: The use of topic initial elicitors. In J. M. Atkinson \& J. Heritage (Eds.), Structures of Social Action: Studies in Conversation Analysis (pp. 167-190). Cambridge: Cambridge University Press. (1985). Topic nomination and topic pursuit. Human Studies, 8, 3-55.

CHAFE, W. (1994). Discourse, Consciousness and Time. Chicago: University of Chicago Press.

CORNISH, F. (1999). Anaphora, Discourse and Understanding. Oxford: Clarendon Press.

COULON, A. (1993). L'ethnométhodologie ( $3^{\text {rd }}$ ed. ed.). Paris: PUF.

ERICKSON, F., \& SHULTZ, J. (1981). When is a context? Some issues and methods in the analysis of social competence. In J. L. Green \& C. Wallat (Eds.), Ethnography and Language in Educational Settings (pp. 147-160). Norwood NJ: Ablex.

FORD, C. E. (1993). Grammar in Interaction: Adverbial Clauses in American English Conversation. Cambridge: Cambridge University Press.

FORNEL, M. de. (1988). Constructions disloquées, mouvement thématique et organisation préférentielle dans la conversation. Langue Française, 78, 101-123.

GARFINKEL, H. (1967): Studies in Ethnomethodology. Englewood Cliffs, N.J., Prentice-Hall.

GARFINKEL, H., \& SACKS, H. (1970). On formal structures of practical actions. In J. D. McKinney \& E. A. Tiryakian (Eds.), Theoretical Sociology (pp. 337-366). New York: Appleton-Century Crofts.

GIVON, T. (1992). The grammar of referential cohenrece as mental processing instructions. Linguistics, 30, 5-55.

GOODWIN, C. (1979). "The interactive construction of a sentence in natural conversation", in: Psathas, G. (Ed.), Everyday Language: Studies in Ethnomethodology. New York, Irvington Publishers, 97-121. (1987). Forgetfulness as an interactive resource. Social Psychology Quarterly, 50-2, 115-130.

GREATBATCH, D. L. (1986). Aspects of topical organisation in news interviews: the use of agenda shifting procedures by interviewees. Media, Culture and Society, 8, 441-455.

GÜLICH, E. (1990). Pour une ethnométhodologie linguistique. Description de séquences conversationnelles explicatives. In M. Charolles \& S. Fisher \& J. Jayez (Eds.), Le Discours: représentations et interprétations (pp. 71-109). Nancy: Presses Universitaires de Nancy.

GÜLICH, E., MONDADA, L. (à paraître). "Analyse conversationnelle”, in G. Holtus, M. Metzeltin, C. Schmitt (eds.), Lexikon der Romanistischen Linguistik, Tübingen, Niemeyer.

HAVE, P. ten (1998). Doing Conversation Analysis. A Practical Guide. London: Sage.

HERITAGE, J. (1984). Garfinkel and Ethnomethodology. Cambridge: Polity Press.

(1992). L'ethnométhodologie: une approche procédurale de l'action et de la communication. Réseaux, 50, 89-131.

HERITAGE, J., \& WATSON, D. R. (1979). Formulations as conversational objects. In G. Psathas (Ed.), Everyday Language: Studies in Ethnomethodology (pp. 123-162). New York: Irvington.

HUDELOT, C. (1988). "Gestion de la différence dans le dialogue adulte-enfant et entre enfants", Cahiers d'Acquisition et de Pathologie du Langage, 3.

HUTCHBY, I., \& WOOFFITT, R. (1998). Conversation Analysis: Principles, Practices and Applications. Oxford: Polity Press. 
JEFFERSON, G. (1978). Sequential aspects of storytelling in conversation. In J. Schenkein (Ed.), Studies in the Organization of Coversational Interaction (pp. 219-248). New York: Academic Press.

(1993). Caveat speaker: preliminary notes on recipient topic-shift implicature. Research on Language and Social Interaction, 26(1), 1-30.

KALLMEYER, W., \& SCHUTZE, F. (1976). Konversationsanalyse. Studium Linguistik, 1, 1-28.

JUBRAN, C.C.S.; URBANO, H.; KOCH, I.G.V. et alii (1992). "Organização tópica da conversação". In: R. ILARI (org.). Gramática do Português Falado, vol.2: Níveis de Análise Lingüística. Campinas: Edunicamp/Fapesp, 357-479.

LAMBRECHT, K. (1994). Information Structure and Sentence Form. Cambridge: CUP.

LERNER, G. H. (1991). “On the syntax of sentence-in-progress”, Language in Society, 20, 441-458.

LEVINSON, S.C. (1983). Pragmatics, Cambridge, CUP.

MARCHUSCHI, L.A. (1998). Análise da conversaçao. São Paulo: Atica.

MAYNARD, D. (1980). Placement of topic changes in conversation. Semiotica, 30, 263-290.

MONDADA, L. (1994). Verbalisation de l'espace et fabrication du savoir: Approche linguistique de la construction des objets de discours, Lausanne: Université de Lausanne.

- (1995a). Introduction: pour une approche des formes linguistiques dans les dynamiques interactionnelles. In L. Mondada (Eds.), Formes linguistiques et dynamiques interactionnelles, Actes du colloque de Lausanne, Cahiers de l'ILSL no 7 (pp. 1-18).

(1995b). La construction interactionnelle du topic. In L. Mondada (Eds.), Formes linguistiques et dynamiques interactionnelles, Actes du colloque de Lausanne, Cahiers de l'ILSL no 7 (pp. 111-135).

(1999). L'organisation séquentielle des ressources linguistiques dans l'élaboration collective des descriptions. Langage et société, 89, 9-36.

. (2000a). Grammaire-pour-l'interaction et analyse conversationnelle. In A.-C. Berthoud \& L. Mondada (Eds.), Modèles du discours en confrontation (pp. 23-42). Berne: Lang.

(2000b). La construction du savoir dans les discussions scientifiques. Apports de la linguistique interactionnelle et de l'analyse conversationnelle à la sociologie des sciences", Revue Suisse de Sociologie 26/3, 2000, 615-36.

. (2001). Linguistica interaccional, Discurso y Sociedad, $3 / 3$.

(2001). (à paraître. Pour une approche conversationnelle des objets de discours. Proceedings do II Congresso Internacional da ABRALIN, Fortaleza, 14-16.3.

MONDADA, L., \& DUBOIS, D. (1995). Construction des objets de discours et catégorisation: une approche des processus de référenciation. Tranel (Travaux Neuchâtelois de Linguistique), 23, 273-302.

MONDADA, L., \& ZAY, F. (1999). Le rôle des parenthèses dans les opérations sur les objets de discours: approche syntaxique, thématique et interactionnelle. In J. Verschueren (Ed.), Pragmatics in 1998. Selected Papers from the 6th International Pragmaticss Conference, vol. 2 (pp. 396-411). Antwerp: IPrA.

NOFSINGER, R. E. (1988/89). Let's talk about the record: Contending over topic redirection in the Rather/Bush interview. Research on Language an Social Interaction, 22, 273-292.

OCHS, E., SCHEGLOFF, A., THOMPSON, S. A. (Eds.), Interaction and Grammar. Cambridge, Cambridge University Press.

ONO, T./THOMPSON, S. A. (1995). "The dynamic nature of conceptual structure building: Evidence from conversation", in: Goldberg, A. (Ed.), Conceptual Structure, Discourse and Language. Cambridge, Cambridge University Press, 105-139. 
ORLETTI, F. (1989). Topic organization in conversation. International Journal of the Sociology of Language, 76, 75-85.

PSATHAS, G. (1995). Conversation analysis: The Study of Talk-in-Interaction. London: Sage.

SACKS, H. (1992 [1964-72]). Lectures on Conversation (2 Vols.). Oxford, Basil Blackwell. (1972). An initial investigation of the usability of conversational materials for doing sociology. In

D. Sudnow (Ed.), Studies in Social Interaction (pp. 31-74). New York: Free Press. (1984). Notes on methodology. In J. M. Atkinson \& J. Heritage (Eds.), Structures of Social Action. Cambridge: Cambridge University Press, 21-27. (1992). Lectures on Conversation, Oxford: Blackwell, 2 voll.

SACKS, H., \& SCHEGLOFF, E. A. (1979). Two Preferences in the Organization of Reference to Persons and Their Interaction. In G. Psathas (Ed.), Everyday Language: Studies in Ethnomethodology (pp. 1521). New York: Irvington Publishers.

SACKS, H., SCHEGLOFF, E. A., JEFFERSON, G. (1974). "A simplest systematics for the organization of turn-taking for conversation”, Language, 50, 696-735.

SCHEGLOFF, E. A. (1986). The routine as achievement. Human Studies, 9, 111-151. . (1990). On the organization of sequences as a source of "coherence" in talk-in-interaction. In D. Dorval (Eds.), Conversational Organization and Its Development (pp. 51-77). Norwood NJ: Ablex. (1990). On the organization of sequences as a source of "coherence" in talk-in-interaction. In D. Dorval (Ed.), Conversational Organization and Its Development (pp. 51-77). Norwood NJ: Ablex.

SCHEGLOFF, E. A., \& SACKS, H. (1973). Opening up closings. Semiotica, 8, 289-327.

SCHEGLOFF, E. A., JEFFERSON, G., \& SACKS, H. (1977). The Preference for self-correction in the organization of repair in conversation. Language, 53, 361-382.

SCHENKEIN, J. (1978). Introduction, in J. Schenkein (ed.), Studies in the Organization of Conversational Interaction. New York: Academic Press.

SELTING, M. (1995). Der "möglische Satz" als interaktiv relevante syntaktische Kategorie. Linguistische Berichte, 158, 298-325.

TERASAKI, A. (1976). Pre-Announcement Sequences in Conversation (Manuscript). 